\title{
ORIGINAL CONTRIBUTION Artificial Neural Networks and Wireless Network Technology: A Review of Literature
}

\author{
Abdul Basit ${ }^{1}$, Noor un Nisa ${ }^{2 *}$ \\ 1,2 Bath Spa University Academic Center Rak, Ras al Khaimah, UAE
}

\begin{abstract}
The research paper is an introduction to the artificial neural networks and wireless network by using sensor technology. There are myriads types of neural networks which are used in computer network and wireless network technology. Neural networks explain and give the proper demonstration for the applications of neural networks and wireless network. The artificial network and wireless network have strong connection and can be investigated and explained properly. There are also some models which are called mathematical models which can explain and demonstrate the artificial and wireless networks in detail. There are many organizations which are using artificial neural networks by using sensor technology for wide range of purpose. A new technology ZigBee introduced in artificial networks and wireless networks by evolving the new protocol system based on meter reading. Artificial neural networks and wireless network along database management system have great importance in revolutionizing the world in artificial and wireless technology by using different wireless sensors, RF chip, and fusion of RFID system along with nodes of ZigBee technology.
\end{abstract}

Index Terms - Artificial Neural Networks, Wireless Network Technology, ZigBee Technology, RFID

Received: 29 July 2020; Accepted: 19 September 2020; Published: 18 December 2020

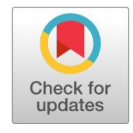

(C) 2020 JITDETS. All rights reserved.

\section{INTRODUCTION}

\section{A. Artificial Neural Networks and Wireless Network Technology}

Artificial neural network can be applied to various fields and has very broad range of tasks in many different disciplines. The main focus is on those areas which are very close to the potential networking system of neural networks and wireless network system. There are many similarities of neural network and human body structure network which detects different types of errors and sort out the problem and errors very carefully. The neural network applications have existing problems in different fields [1]. There are some applications which give the arbitrary solution. There are some same characteristics which can categories neural networks classification in different problems. The main ability of neural network structure is to perform different universal functions like ability to form and decide complex decisions on the bases of generalizations [2]. There are some capabilities of importance in virtually models of neural networks which can be derived from observed behavior and constraints. Artificial neural network system is information processing system which designed and inspired like as the nervous system [3]. This works as like human brain processes the information for different types of inputs in biological way. The main key element is for this system, which is called information system of different types networking system [4]. This system comprises over on many highly interconnected elements like neurons. These elements solved different problems. The ANN and wireless technology system configure different specific application and problems according to the recognition of pattern system and different types of classification of data. This sys-

\footnotetext{
${ }^{*}$ Corresponding author: Noor un Nisa

${ }^{\dagger}$ Email: noor@bathspa.ae
}

tem exists between the biological and synaptic connections of neurons. In wireless technology, computer network and data network based on different systems like different types of wires, switches, routers and wireless systems. In computer network system, all the computer systems and network devices pass through data from different states to other devices for connectivity and sharing of data [5]. The connections are established with wires and wireless technology system with devices of networks. These connections are established with nodes, clients or server computers and dedicated servers are also used for sharing, sending and receiving of data.

\section{B. Contributions to Neural and Wireless Network Technology}

This paper offers wide range of different contributions to the neural network and wireless network technology. The research is based on neural network system and wireless network technology [6]. It is complete view of neural network and wireless network technology. Once the protocol system for the wireless network and neural network are set for the monitoring, the system becomes easy to monitor the setup. The paper represents the neural network, wireless technology and hard structure of the system to monitor with sensors. There are different types of solutions are introduced in the paper for the monitoring of the network and detecting the system properly if errors are found [7]. 


\section{HISTORICAL BACKGROUNDS ARTIFICIAL NEURAL NETWORKS AND WIRELESS NETWORK TECHNOLOGY}

In present time the neural network system is appeared as recent development system as simulation. But this networks field established before the invention of computers and this change gave a big setback in different times and eras. There are many advances which are developed and boosted by computers emulations in different times. This period is very tough but becomes as disrepute with frustration $[8,9]$. That was the time when funding support was just minimal or no funding some time. The professional support was not according to best level. The Pioneers developers are able to develop proper technology which can remove the limitations from the networks. This neural network also has history related with the development of neurophysiologist [10]. These devices are made to talk with each other for communication purpose. Early system was used to send telegraphs and telephone has invented from these types of things for communication. Early devices for communications were different and complicated for passing the data but now days the system is completely changed [11]. The developers have connected the computers system in such a way that these computers can use different resources, sharing of data and transferring of data worldwide in different formats. The new system was introduced as communication protocol which is known as data packet switching with number of applications like voice transmissions and other protocols [12]. These networks are providing the main basis for the ARPANET which is the main base of the present networking system.

\section{A. Neural Network and Wireless Network Protocol}

In modern era, Wireless Local Area Network (WLAN) technology and protocols are becoming popular in connectivity with many different types of devices. The latest protocols are Bluetooth Home RF and ultra wideband technology [13]. These protocols are developed in last seven to ten years. By using these technologies, these technologies remove the wires fro devices. The location can be found without wires with the help of protocols. The supporting companies are developing an infrastructure called Device Centric Services (DCS). These services provide the set of monitoring the applications. This also includes automated meter reads for monitoring the readings. This latest infrastructure currently has support to all other devices [14].

\section{B. Neural Networks and Wireless System Networks Properties}

The structure of neural networks is remarkable and has ability to maintain complicated data. This data can be used to extract different patterns and has ability to detect different trends which are complex and be noticed by the humans or by the computer systems techniques [15]. A well trained artificial neural network can be as expert I different information systems and many other interested projections with suitable system.

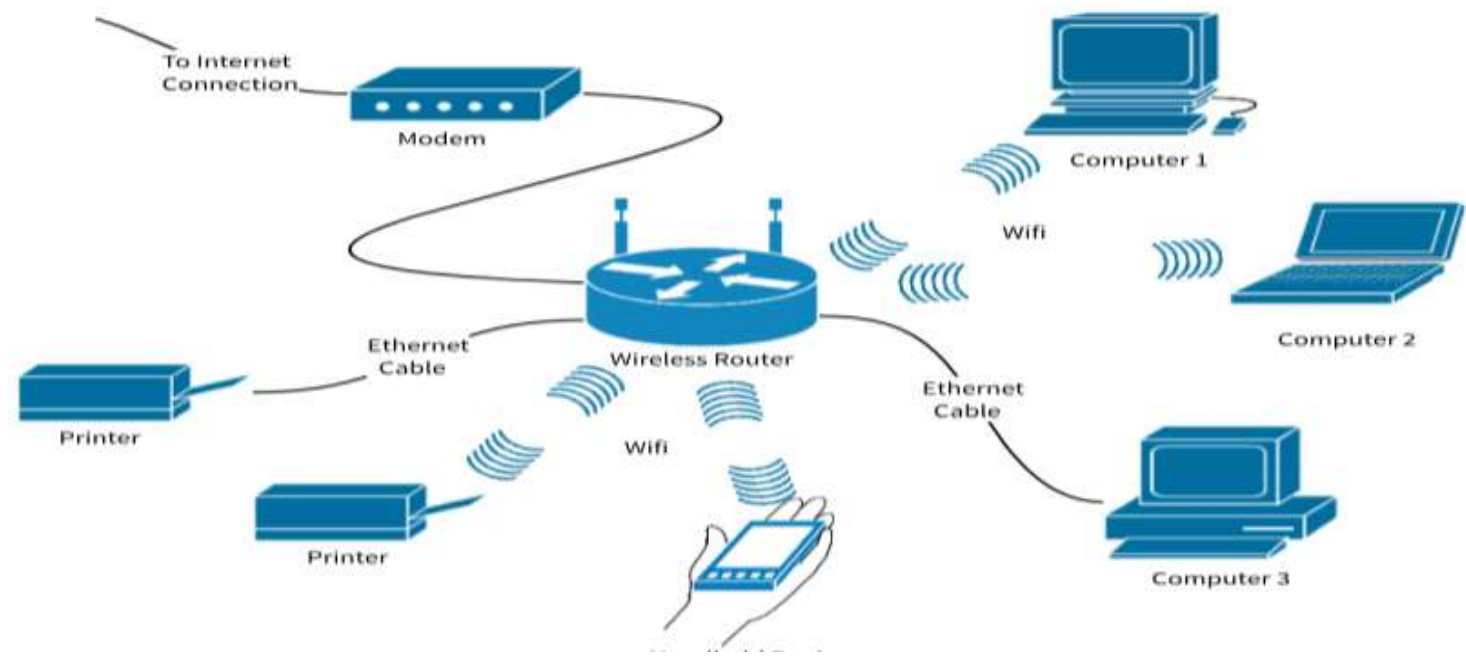

Fig. 1. Communications of devices with wireless networking

\section{Pulse Amplitude Modulation in Network and Wireless Network Sys- tem (PAM)}

PAM is a modulation method and technique which can transmit a symbol of amplitudes to the transmitted signals for the reassembling of digital signals [16]. This technique is very simple and similar to the analog system which can monitor the whole system properly. In digital system, there are some numbers which participate in the network. A PAM signal can map of certain bits.

\section{Adaptive Learning of Neural Network}

The ability of the network is to learn about tasks how to perform in different initial experiences of the network system. The different users and people can efficiently use networking system to communicate the other user by different sources and channels to send and receive emails and transfer data from one place to another place. In this situation authorize people can access the data from different computers [17]. (TCP)

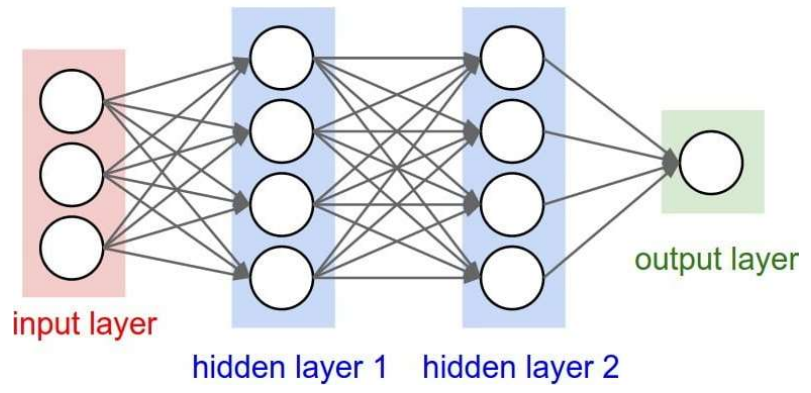

Fig. 2. Description of pattern in neural network 


\section{ORGANIZED STRUCTURES OF NEURAL NETWORK AND WIRELESS SYSTEM}

Neural network can create and organized the representation of data and information system decoders and learning time. Users and people have very easy access to the communication sources because of different networking and channels system. These systems easily can send and receive data from networking links [18].

\section{A. Pulse Position Modulation (PPM) I Network System}

This is another technique which is similar to the PAM but little bit different. In this technique magnitude is not adjusted [19]. Pulse defines the symbol and position of the different symbols. PPM is used in optical systems and transfer signals as transmission of segments. This can break the transmission segments into different communicational parts. This value can signify different symbols in network. (TDMA)

\section{B. Real Time Operation in Neural Networks and Wireless System}

The neural system which is being designed for the computational results and maintaining the network system if problems happened will be resolved automatically [20]. There are different types of information sharing system which can use authorization for sharing and receiving data from different user. There is different types of information which can be distributed and submitted on different sources. Authorization is very important in all areas of networking.

\section{Neural network and Fault Tracing with Coding}

If network system or wireless network system collapsed with corresponding to the performance becomes hard to find out the problems but network technologies retained and maintain the system properly [21, 22].

\section{Neural Computer Networks and Wireless Communication System}

Users and people can use different networks and do communication effectively via different channels like emails, messaging system, video conference and telephone calls. All the channels are handled and controlled by networking system [23].

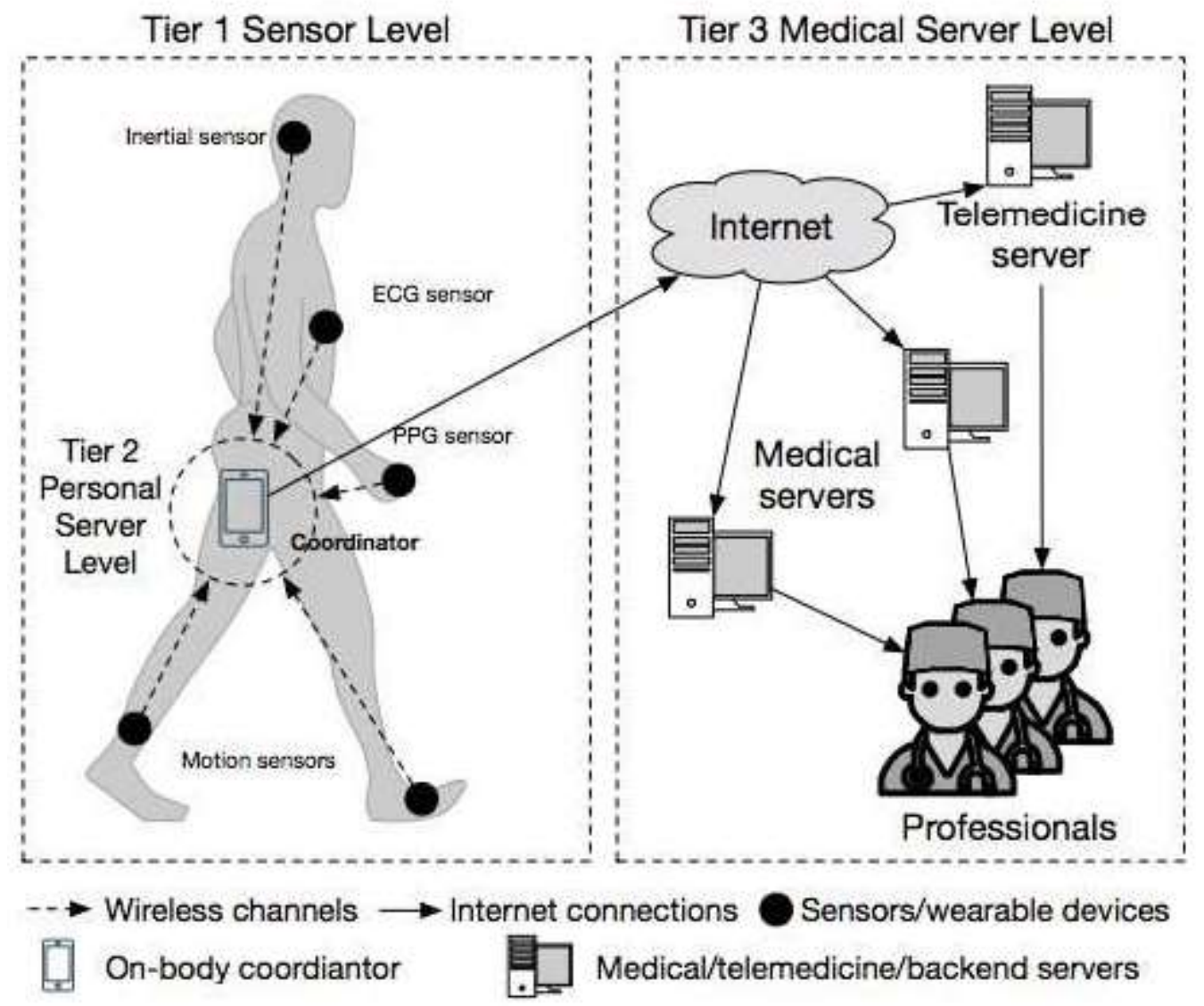

Fig. 3. Neural network and sensors

\section{E. Network detection Frequency Shift Keying in Neural and Wireless Network System (FSK)}

FSK is digital modulation method and technique which can be similar in frequency and modulation of FM. This supports to analog devices. But this is the main difference between FSK and FM. Each digital symbol has different frequencies of 101 for FSK. This is the map which can specify the frequency properly. The main and big advantage is that this FSK assigns the symbols in network [24].

F. Neural system and Orthogonal Frequency Division Multiplexing in Network and Wireless System of Neural Network (OFDM)

The main task of OFMD is to divide the modulation parts in band- 
width into smaller bandwidth of modulation. This can carry the frequencies that close to the orthogonally in the network system. This bandwidth of OFDM consist single set of transmitted data. This can transmit the data into different smaller parts [25].

\section{G. Neural network and wireless techniques for Phase Shift Keying (PSK)}

This is another technique that is similar to the modulation phase that communicates with the system in modulation. There are finite set of phases that maps in specific bit rate [26]. This binary map bit key can map to the keys to the zero value. There are two main symbols of BPSK.

\section{H. Artificial Neural Networks and Wireless Network Comparison of Conventional Computers}

Neural network can deal with different and different approaches to sort out the problems and networking system. Conventional computers are used I networking and artificial intelligence. Computers receive the instructions to handle different types of instructions so that machine will execute the instructions properly and process data [27]. There is problem in doing the specific steps and execute the steps in different ways. Sometimes computer can follow the steps and execute the instructions properly. There are some conventional computers which can support the systems in networks. The computers can sort different conflict issues between the systems and the other servers which are connected with that [28]. There are some restricted problems which can sort out the problems quickly. There are some computers which can be more useful and can better handle the network security and structure of the networking system. The problems with networking and the servers are the main problem of lot of users at the same time [29]. The neural network can process all the information as our human brain is processing the information.

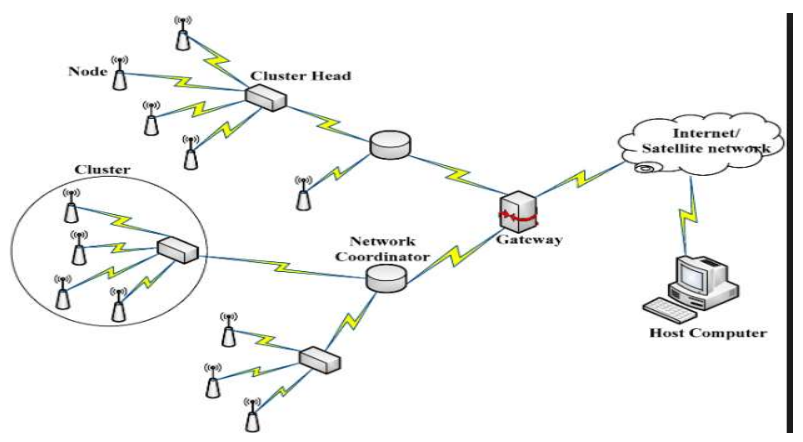

Fig. 4. Networking with servers and WAN

\section{G. In Network Carrier Sense Multiple Access With Collision Avoidance and Techniques (CSMA/CA)}

There are many different types of protocols and MAC protocols which can develop the CSMA/CA system to avoid from collision in the network. In this research paper only general idea is used for the CSMA/CA. CSMA/CA and MAC has three main and major problems. There is one main problem called hidden node [30]. There are also some other problems called exposed node problem, capture problem and hidden problem. There are two main techniques which are used in MAC and CSMA/CA. these two techniques are out of band structure of the network. This technique is suitable and can remove the hidden node problems and effect of this can expose the numbers of nodes [31]. When data is transmitted in network this technique can avoid the CSMA/CA collision problem. This technique has large amount of overheads but it can reduce the effect and problem of all three channels of protocols. There are certain levels of finding the CSMA/CA in networking [32].
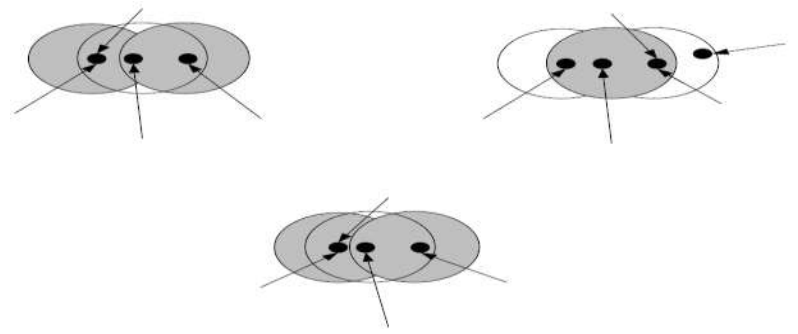

Fig. 5. Network protocols

This information can be loaded on to our mind but he criteria is same for our computers for processing of data and information. Neural network can be learn and wireless network by technology. In this research, three companies are selected according to requirement engineering models. These three models are actually selected because of their structural difference. There structural differences are linear and linear iterations between activities and iterative. Requirement engineering models are depicted with linear and incremental models. Requirement engineering models are commonly used requirement engineering models activities and elicitation analysis. (TKIP) These requirement engineering models are combined under these headings but still follow similar linear transition under this model. There are two models are selected under this research. (TPC) These models state that these activities are in this model performed iterative structure. Before using requirement engineering models current RE practices were giving the different views as an indication of common activities and requirement engineering models sequence. 


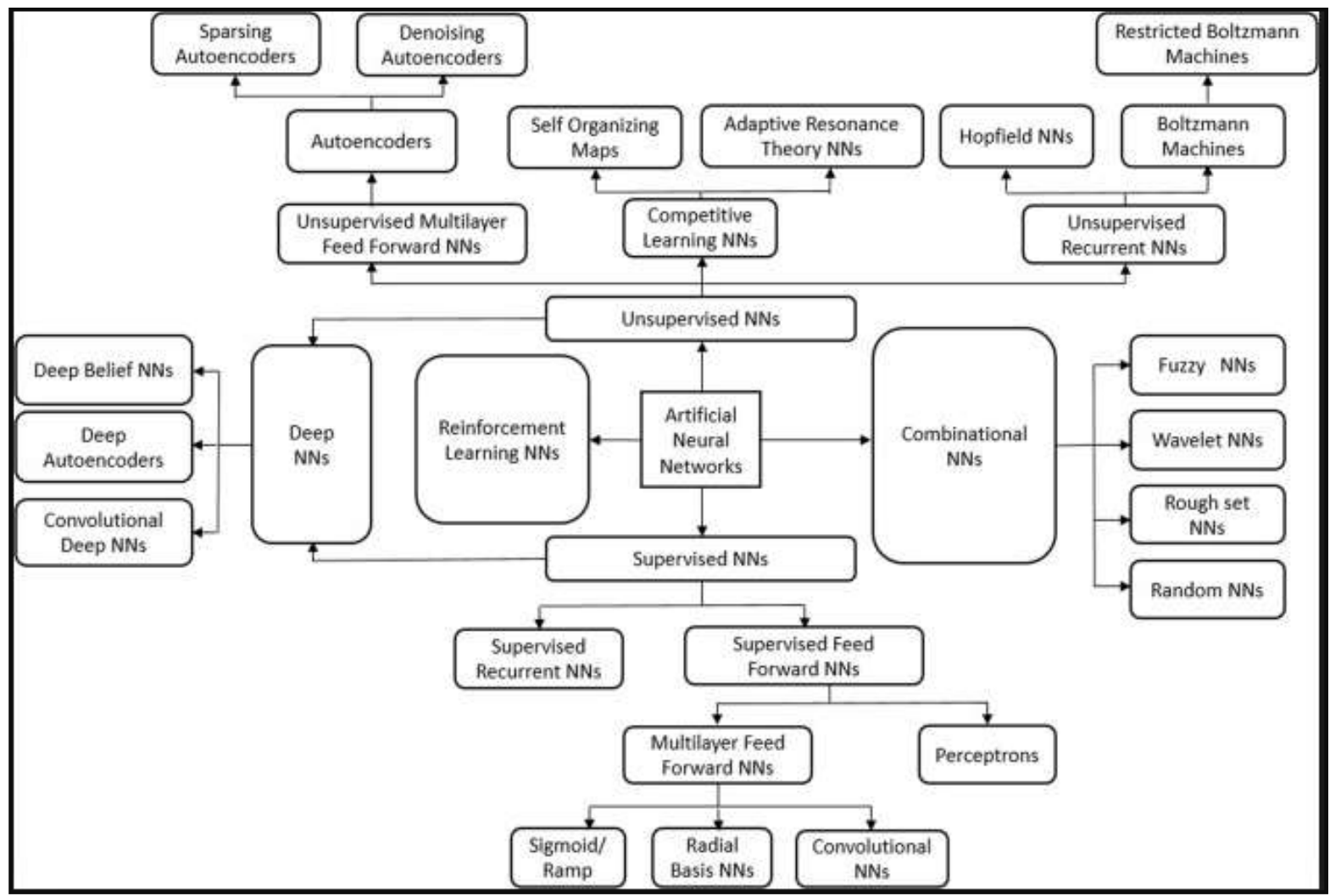

Fig. 6. Network structure

But these models can be different and sometimes conflicting because of their structural nature ranging from linear to incremental cyclical structure. According to previous studies, requirement engineering models different companies use different models. Requirement engineering models are used then further complications are raised with the idea which that process models are situation dependent and also affected by customer supplier chain model. The product on maturity level will reach due to requirement engineering models and disciplinary involvement. Requirement engineering model is the process of eliciting individual stakeholders participate. They can be use as to perform specific tasks and these tasks can be done in neural networks in different ways. The examples which are related to the networks and security can handle the data properly. But the main issue is that how to sort the problem and in this way operation can become unpredicted. Some computers can use the cognitive approach for problem solving. The problem stated can be must know and clear. These instructions can be highlighted and stated in different and small instructions. These instructions can be later converted into high level programming language and then it will be converted easily to machine code. These machines are predicted and take to the right direction and if these things having problem then it goes to wrong direction because of hardware fault. The main difference of networks in neural and conventional algorithms is of completion of different machines hardware structure. These are the tasks which are more helpful in algorithmic approach like arithmetic structure. The neural networks can easily handle the large number of tasks.
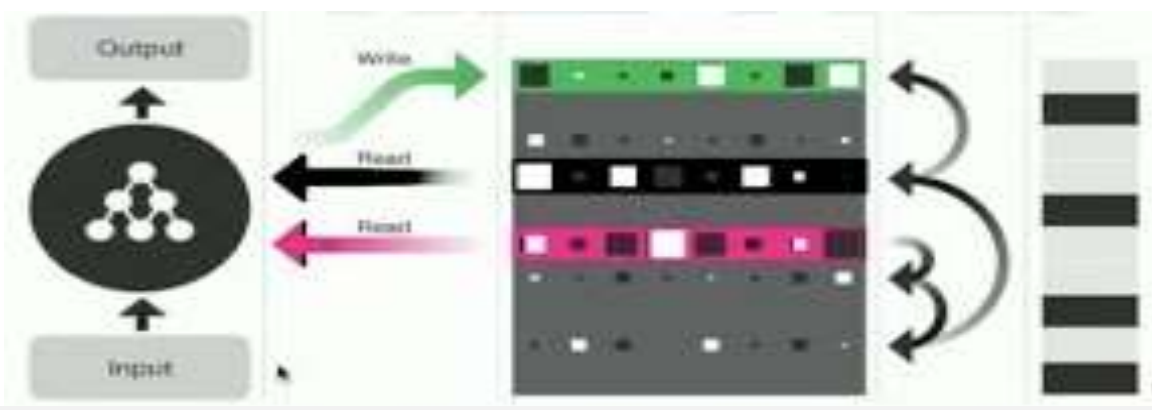

This consists of a neural network that can read from and write to an external memory analogous to the random-access memory in a conventional computer.

Fig. 7. Neural network sensors communication 


\section{CONCLUSION}

There are many companies which are using neural network system. These companies make the system powerful and flexible. There is no need to devise algorithm to perform a specific task. There are no more tasks to perform properly. There are also many tasks well suited for different algorithms. There are different mechanisms for the division of number of tasks. These tasks can be suited and much better for real time. They have good response and architectural results. There is parallel system run with the support of real time. Neural networks can also contribute to those areas in which research is done like neurology and other departments. They are regularly using different models of models parts in living organisms. The internal mechanism can be easily investigated. The neural network is most inspective and network created system which deals different sensors. The neural network system can automatically detect sensors and pass the data to other nodes of the server. Most exciting part is conscious area of network which can produce mechanical property. So the finally I would like to explain more terms and details of the network system which can produce huge network system in wireless and other network servers. These networks also promote the fuzzy logic and other related networks.

\section{References}

[1] Deloitte and MedTech Europe. (2020) The socio-economic impact of $\mathrm{AI}$ in healthcare. [Online]. Available: https://bit.ly/319jeUE

[2] M. Haseeb, L. W. Mihardjo, A. R. Gill, K. Jermsittiparsert et al., "Economic impact of artificial intelligence: New look for the macroeconomic assessment in Asia-Pacific region," International Journal of Computational Intelligence Systems, vol. 12, no. 2, pp. 1295-1310, 2019. doi: https://doi.org/10.2991/ijcis.d.191025.001

[3] V. Van Roy et al., "Ai watch-national strategies on artificial intelligence: A European perspective in 2019," Joint Research Centre (Seville site), Seville, Spain, Working paper JRC119974, 2020.

[4] E. Garfield, "Citation analysis as a tool in journal evaluation," Science, vol. 178, no. 4060, pp. 471-479, 1972.

[5] A. Van Raan, "The use of bibliometric analysis in research performance assessment and monitoring of interdisciplinary scientific developments," TATuP-Zeitschrift für Technikfolgenabschätzung in Theorie und Praxis, vol. 12, no. 1, pp. 20-29, 2003. doi: https://doi.org/ 10.14512/tatup.12.1.20

[6] G. Abramo, C. D’Angelo, and F. Pugini, "The measurement of Italian universities' research productivity by a non parametric-bibliometric methodology," Scientometrics, vol. 76, no. 2, pp. 225-244, 2008. doi: https://doi.org/10.1007/s11192-007-1942-2

[7] J. Mokhnacheva and I. Mitroshin, "Nanoscience and nanotechnologies at the Moscow domain: A bibliometric analysis based on web of science (Thomson Reuters)," Information Resources of Russia, vol. 6, pp. 17-23, 2014.

[8] K. Debackere and W. Glänzel, "Using a bibliometric approach to support research policy making: The case of the Flemish BOF-key," Scientometrics, vol. 59, no. 2, pp. 253-276, 2004. doi: https://doi.org/ 10.1023/b:scie.0000018532.70146.02

[9] R. Suryanita, H. Maizir, and H. Jingga, "Prediction of structural response based on ground acceleration using artificial neural networks," International Journal of Technology and Engineering Studies, vol. 3, no. 2, pp. 74-83, 2017. doi: https://doi.org/10.20469/ijtes.3. 40005-2
[10] H. F. Moed, "The effect of "open access" on citation impact: An analysis of ArXiv's condensed matter section," Journal of the American Society for Information Science and Technology, vol. 58, no. 13, pp. 2047-2054, 2007. doi: https://doi.org/10.1002/asi.20663

[11] T. U. Daim, G. R. Rueda, and H. T. Martin, “'Technology forecasting using bibliometric analysis and system dynamics," in A Unifying Discipline for Melting the Boundaries Technology Management, Portland, OR, 2005.

[12] T. U. Daim, G. Rueda, H. Martin, and P. Gerdsri, "Forecasting emerging technologies: Use of bibliometrics and patent analysis," Technological Forecasting and Social Change, vol. 73, no. 8, pp. 981-1012, 2006. doi: https://doi.org/10.1016/j.techfore.2006.04.004

[13] T. Inaba and M. Squicciarini, ICT: A new taxonomy based on the international patent classification. Paris, France: OECD, 2017.

[14] L. Egghe, "Dynamic h-index: The Hirsch index in function of time," Journal of the American Society for Information Science and Technology, vol. 58, no. 3, pp. 452-454, 2007. doi: https://doi.org/10.1002/ asi. 20473

[15] R. Rousseau and F. Y. Ye, "A proposal for a dynamic h-type index," Journal of the American Society for Information Science and Technology, vol. 59, no. 11, pp. 1853-1855, 2008. doi: https://doi.org/10. 1002/asi.20890

[16] J. S. Katz, "Scale-independent indicators and research evaluation," Science and Public Policy, vol. 27, no. 1, pp. 23-36, 2000. doi: https: //doi.org/10.3152/147154300781782156

[17] A. F. Van Raan, "Fatal attraction: Conceptual and methodological problems in the ranking of universities by bibliometric methods," Scientometrics, vol. 62, no. 1, pp. 133-143, 2005. doi: https://doi.org/ 10.1007/s11192-005-0008-6

[18] R. I. Muhamedyev, R. M. Aliguliyev, Z. M. Shokishalov, and R. R. Mustakayev, "New bibliometric indicators for prospectivity estimation of research fields," Annals of Library and Information Studies, vol. 65, p. 62-69, 2018.

[19] B. Copeland. (2020) Artificial intelligence. [Online]. Available: https://bit.ly/3mqm5If

[20] M. Michael. (2016) Artificial intelligence in law: The state of play 2016. [Online]. Available: https://bit.ly/2WI70s]

[21] G. Nguyen, S. Dlugolinsky, M. Bobák, V. Tran, Á. L. García, I. Heredia, P. Malík, and L. Hluchỳ, "Machine learning and deep learning frameworks and libraries for large-scale data mining: A survey," Artificial Intelligence Review, vol. 52, no. 1, pp. 77-124, 2019. doi: https://doi.org/10.1007/s10462-018-09679-z

[22] N. Ugtakhbayar, B. Usukhbayar, S. H. Sodbileg, and J. Nyamjav, "Detecting tcp based attacks using data mining algorithms," International Journal of Technology and Engineering Studies, vol. 2, no. 1, pp. 1-4, 2016. doi: https://doi.org/10.20469/ijtes.2.40001-1

[23] J. A. Cruz and D. S. Wishart, "Applications of machine learning in cancer prediction and prognosis," Cancer Informatics, vol. 2, pp. 59-77, 2006. doi: https://doi.org/10.1177/117693510600200030

[24] R. Miotto, F. Wang, S. Wang, X. Jiang, and J. T. Dudley, "Deep learning for healthcare: Review, opportunities and challenges," Briefings in Bioinformatics, vol. 19, no. 6, pp. 1236-1246, 2018. doi: https: //doi.org/10.1093/bib/bbx044

[25] P. J. Ballester and J. B. Mitchell, “A machine learning approach to predicting protein-ligand binding affinity with applications to molecular docking," Bioinformatics, vol. 26, no. 9, pp. 1169-1175, 2010. doi: https://doi.org/10.1093/bioinformatics/btq112 
[26] M. S. Mahdavinejad, M. Rezvan, M. Barekatain, P. Adibi, P. Barnaghi, and A. P. Sheth, "Machine learning for internet of things data analysis: A survey," Digital Communications and Networks, vol. 4, no. 3, pp. 161-175, 2018. doi: https://doi.org/10.1016/j.dcan.2017.10.002

[27] C. R. Farrar and K. Worden, Structural Health Monitoring: A Machine Learning Perspective. New York, NY: JohnWiley \& Sons, 2012.

[28] J. Lai, J. Qiu, Z. Feng, J. Chen, and H. Fan, "Prediction of soil deformation in tunnelling using artificial neural networks," Computational Intelligence and Neuroscience, vol. 2016, pp. 1-16, 2016. doi: https://doi.org/10.1155/2016/6708183

[29] K. G. Liakos, P. Busato, D. Moshou, S. Pearson, and D. Bochtis, "Machine learning in agriculture: A review," Sensors, vol. 18, no. 8, pp. 1-29, 2018. doi: https://doi.org/10.3390/s18082674
[30] V. Tatarinov, A. Manevich, and I. Losev, "A system approach to geodynamic zoning based on artificial neural networks," Gornye Nauki i Tekhnologii= Mining Science and Technology (Russia), no. 3, pp. 14-25, 2018.

[31] C. Clancy, J. Hecker, E. Stuntebeck, and T. O'Shea, "Applications of machine learning to cognitive radio networks," IEEE Wireless Communications, vol. 14, no. 4, pp. 47-52, 2007. doi: https://doi.org/10.1109/ MWC.2007.4300983

[32] N. M. Ball and R. J. Brunner, "Data mining and machine learning in astronomy," International Journal of Modern Physics D, vol. 19, no. 07, pp. 1049-1106, 2010. doi: https://doi.org/10.1142/ S0218271810017160 